\title{
Laurence DAHAN-GAIDA (dir.), Circulation des savoirs et reconfiguration des idées. Perspectives croisées France-Brésil
}

\section{Thomas Vercruysse}

\section{(2) OpenEdition Journals}

Édition électronique

URL : http://journals.openedition.org/ress/3688

DOI : $10.4000 /$ ress.3688

ISBN : 1663-4446

ISSN : $1663-4446$

\section{Éditeur}

Librairie Droz

\section{Édition imprimée}

Date de publication : 15 mai 2017

Pagination : 275-278

ISSN : 0048-8046

Référence électronique

Thomas Vercruysse, «Laurence DAHAN-GAIDA (dir.), Circulation des savoirs et reconfiguration des idées. Perspectives croisées France-Brésil », Revue européenne des sciences sociales [En ligne], 55-1 | 2017, mis en ligne le 14 novembre 2016, consulté le 19 janvier 2021. URL : http://journals.openedition.org/ress/ 3688 ; DOI : https://doi.org/10.4000/ress.3688

Ce document a été généré automatiquement le 19 janvier 2021.

(c) Librairie Droz 


\title{
Laurence DAHAN-GAIDA (dir.), Circulation des savoirs et reconfiguration des idées. Perspectives croisées France-Brésil
}

\author{
Thomas Vercruysse
}

\section{RÉFÉRENCE}

Laurence DAHAN-GAIDA (dir.), 2016, Circulation des savoirs et reconfiguration des idées. Perspectives croisées France-Brésil, Villeneuve d'Asq, Presses Universitaires du Septentrion, $412 \mathrm{p}$.

1 Laurence Dahan-Gaida est une spécialiste chevronnée des transferts de savoirs, de leurs migrations et de leurs reprises dans un espace culturel donné ainsi que l'une des figures majeures de la méthode d'analyse littéraire que l'on appelle, à la suite de Michel Pierssens, l'épistémocritique (voir, de ce dernier, Savoirs à l'œeuvre. Essais d'épistémocritique, Presses universitaires de Lille, 1990). L'ouvrage qu'elle a dirigé est le résultat d'une collaboration entre le Centre de recherches interdisciplinaires et transculturelles (CRIT - EA 3224) de l'université de Franche-Comté et le groupe « Histoire intellectuelle, histoire des intellectuels, historiographie » de l'université fédérale du Paraná à Curitiba (Brésil), et s'inscrit à la croisée de deux programmes de recherche, l'un ayant pour objet l'histoire intellectuelle, l'autre la circulation des savoirs. Ce collectif part du principe, notamment validé dans le domaine culturel par les travaux de l'anthropologue et sociologue indo-américain Arjun Appadurai (l'auteur de Modernity at Large, University of Minnesota Press, 1996), que la nation ne saurait constituer l'échelle d'analyse la plus heuristique pour rendre raison de l'histoire intellectuelle. On fait donc ici la part belle à la mobilité des savants, des artistes comme des penseurs et la question de l'histoire nationale tend à s'effacer devant celle d'histoires « croisées ». 
2 Composée de cinq parties («Transferts culturels et circulations transnationales»; « Historicité des savoirs»; « Nouveaux médias, nouvelles interactions »; «Circulation des idées dans les sciences humaines et sociales "; «Du savoir à la fiction et retour »), résolument interdisciplinaire et transnationale, cette entreprise éditoriale réunit les contributions de 21 spécialistes d'histoire, de sociologie, de pédagogie, de musicologie, d'études littéraires, de théâtre, de littérature comparée ainsi que de différentes langues. L'angle d'attaque choisi, contrairement à ce que le sous-titre du volume pourrait laisser penser, ne se cantonne pas au dialogue entre France et Brésil et s'intéresse également à l'apport des pays du Sud (ici ceux de l'Amérique latine) dans l'élaboration contemporaine des savoirs et la modification des rapports de pouvoir (et donc de savoir) qu'elle reflète.

3 Dans son introduction («Savoirs en mouvement. Circulation, percolations, reconfigurations»), Laurence Dahan-Gaida pointe l'émergence du «paradigme de la mobilité » dans l'intégralité des sciences humaines et sociales, émergence poussant à la péremption des notions d'«influence» et de "diffusion" au profit de concepts décrivant des processus multilinéaires comme celui de "réseau » (p. 14), plus à même de peindre les différents transferts de savoir. Elle s'emploie ainsi à distinguer différentes sortes de transferts: la distance comblée peut relier deux moments historiques, auparavant scindés (pour ce qui concerne «la réactualisation des savoirs passés », p. 15), deux cultures ou sous-cultures (dans le cas des phénomènes d'acculturation ou de transculturation), deux niveaux de culture (s'échelonnant de manière verticale : la "culture savante " et la "culture populaire »), deux disciplines voire davantage (la fameuse interdisciplinarité), deux types de discours ou deux textes (interdiscursivité et intertextualité), deux médias ou deux arts (intermédialité ou transmédialité), etc., la liste n'impliquant pas des opérations exclusives les unes des autres mais bien souvent des gestes conjoints.

4 Figurant dans la première partie du livre, l'article d'Helenice Rodrigues da Silva ("Transferts des savoirs, transferts interculturels : méthode et théorie ») remarque que les travaux sur les transferts culturels, suivant d'abord une méthode comparatiste, ont rapidement délaissé le cadre d'analyse des historiographies nationales pour accueillir, à partir du milieu des années 1990, les concepts de "carrefours, d'imbrications, de métissages interculturels » (p.43). Participant d'un renouveau de l'herméneutique, un nombre conséquent de travaux a alors permis d'étoiler les "lieux de la mémoire étrangère » (p.44). Elle cite, par exemple, le cas de l' «histoire croisée » (connected history) aux États-Unis et, plus récemment, en France, la reprise théorique que l'on doit à Michaël Werner (voir notamment l'ouvrage qu'il a dirigé avec Bénédicte Zimmermann, De la comparaison à l'histoire croisée, Éditions du Seuil, 2004). Si elle admet la validité du concept de "transferts culturels ", en particulier pour éclairer l'histoire intellectuelle, elle signale certaines difficultés dans l'application pratique de ce modèle et avance des pistes à suivre pour mieux le mobiliser. Elle insiste à propos sur le fait que "c'est le pôle de la réception [...] qui détermine ce qui mérite d'être importé par les individus et par les groupes qui transportent d'un côté à l'autre de la "frontière" les éléments d'un système " (p. 52). À la lire, on pourrait donc dire que la théorie des «transferts culturels» n'obéit pas au schéma de communication standard (dit "système radio ») émetteur-récepteur, univoque, mais apparaît au fond plus proche celui de la boucle rétroactive, bi-univoque, que l'on trouve aussi bien en cybernétique 
(l'effet feed-back mis en évidence par Norbert Wiener) qu'en herméneutique (que l'on songe à la notion d'horizon d'attente de Hans-Robert Jauss).

Dans son texte ouvrant la cinquième partie ( Transferts entre littérature et savoirs : vers une théorie hétéronome de la littérature ?»), Christine Baron, quant à elle, prend également acte du risque inhérent à cette vectorisation à sens unique qui a trop souvent aimanté les études épistémocritiques depuis l'ouvrage classique de Max Black (Models and Metaphors: Studies in Language and Philosophy, Cornell University Press, 1962), schéma d'après lequel "les sciences migreraient dans la littérature qui les "accommoderait" passivement, en général suivant une finalité didactique de nature explicative (vulgarisation) ou démonstrative (légitimation)» (p. 325). Ce qui retient ici Christine Baron, c'est la façon dont certains champs du savoir, en l'occurrence le droit et l'économie, s'accaparent la théorie littéraire. La notion même de "paradigmes littéraires » est alors interrogée car il paraît délicat de mesurer ce qui la distingue des paradigmes dits «scientifiques ». L'exemple de la «jurisfiction», à mi-chemin entre littérature et droit, encourage, à la suite de François Ost (Raconter la loi, Odile Jacob, 2014), à renoncer au stéréotype d'une répartition des tâches entre la loi et la littérature d'après laquelle la première serait productrice de normes quand la seconde les contesterait: «La littérature est aussi instituante dans la mesure où elle prend appui sur des représentations partagées, et pour faire vite sur un imaginaire du "juste", du "légitime" qui précède notre expérience du droit.»(p.330). Quant à la théorie économique, elle permet notamment de relire à nouveaux frais un classique de la réflexivité littéraire, comme Les Faux Monnayeurs de Gide: "la théorie monétaire devient le medium d'un questionnement poétique; par exemple, la loi de Gresham selon laquelle la monnaie vile circule, la bonne monnaie étant réservée à un usage privé et confidentiel, est l'analogon de la circulation des textes dans le roman et de l'épineuse question de la valeur littéraire que met en scène le roman » (p. 332).

6 Si les contributions précédemment examinées montrent l'obsolescence du modèle de l'emprunt pour rendre compte des transferts culturels, celle de Danielle Follett ( Le néoplatonisme, la poésie et la musique de la nature »), dans la deuxième partie du livre, nourrit l'ambition de renouveler l'histoire des idées. Pour ce faire, elle part de la nonadéquation entre mot et idée : « La non-identité entre mot et idée ne signifie pas que les idées soient des essences supra-historiques se manifestant à travers des mots: elles sont bien plutôt des constructions conceptuelles contingentes et complexes, des produits de l'histoire réelle.» (p.193). Danielle Follett s'attache donc à enquêter sur l'histoire de l'idée de «musique de la nature » dans une optique à la fois comparatiste et diachronique afin de mettre au jour ses implications et ses croisements au cours de ses transferts entre de multiples disciplines (musique, philosophie, religion, sciences, psychologie, littérature), différentes périodes ( $\mathrm{du} \mathrm{XVII}^{\mathrm{e}}$ au XIX ${ }^{\mathrm{e}}$ siècle) et plusieurs pays (Angleterre, États-Unis) en se concentrant sur Cudworth, Newton, Coleridge et Emerson.

7 Cet ouvrage combine une grande richesse dans les domaines convoqués (géographiques et disciplinaires) et une cohérence salutaire dans la méthode, faisant notamment la part belle à l'herméneutique. Gageons qu'il fera école en tramant un tissu de prises utiles pour saisir les mutations idéologiques et épistémologiques d'un monde dont le caractère multipolaire légitime une révision des outils analytiques qui reste, encore, à pleinement acter. 


\section{AUTEURS}

\section{THOMAS VERCRUYSSE}

Université du Luxembourg, IPSE 